Chronic Obstructive Pulmonary Diseases: Journal of the COPD Foundation

\author{
Original Research
}

\title{
Race and Gender Disparities are Evident in COPD Underdiagnoses Across all Severities of Measured Airflow Obstruction
}

A. James Mamary, $\mathrm{MD}^{1}$ Jeffery I. Stewart, $\mathrm{MD}^{1}$ Gregory L. Kinney, $\mathrm{PhD}^{2}$ John E. Hokanson, $\mathrm{PhD}^{2}$ Kartik Shenoy, MD ${ }^{1}$ Mark T. Dransfield, $\mathrm{MD}^{3}$ Marilyn G. Foreman, MD, MS ${ }^{4}$ Gwendolyn B. Vance, $\mathrm{RN}^{1}$ Gerard J. Criner, $\mathrm{MD}^{1}$ for the COPDGene ${ }^{\circledR}$ Investigators

\begin{abstract}
The COPD Genetic Epidemiology (COPDGene ${ }^{\circledR}$ ) study provides a rich cross-sectional dataset of patients with substantial tobacco smoke exposure, varied by race, gender, chronic obstructive pulmonary disease (COPD) diagnosis, and disease. We aimed to determine the influence of race, gender and Global initiative for chronic Obstructive Lung Disease (GOLD) stage on prevalence of prior COPD diagnosis at COPDGene ${ }^{\circledR}$ enrollment. Data from the complete phase 1 cohort of 10,192 participants were analyzed. Participants were non-Hispanic white and African-American, $\geq 45$ years of age with a minimum of 10 pack years of cigarette smoking. Characterization upon enrollment included spirometry, demographics and history of COPD diagnosis determined by questionnaire. We evaluated the effects of race and gender on the likelihood of prior diagnosis of COPD and the interaction of race and GOLD stage, and gender and GOLD stage, as determined at study enrollment, on likelihood of prior diagnosis of COPD. We evaluated the 3-way interaction of race, gender and GOLD stage on prior diagnosis. African-Americans had higher odds of not having a prior COPD diagnosis at all GOLD stages of airflow obstruction versus non-Hispanic whites $(p<0.0001)$. Women had higher odds of having a prior COPD diagnosis at all GOLD stages versus men $(p<0.0001)$. Three-way interaction of race, gender and GOLD stage was not significant. African-Americans were less likely to have prior COPD regardless of the severity of airflow obstruction determined at study enrollment. Women were more likely to have a prior COPD diagnosis regardless of the severity of measured airflow obstruction. Race and gender are associated with significant disparities in COPD diagnosis.
\end{abstract}

Abbreviations: COPD Genetic Epidemiology, COPDGene; chronic obstructive pulmonary disease, COPD; Global initiative for chronic Obstructive Lung Disease, GOLD; African American, AA; non-Hispanic white, NHW; American Thoracic Society, ATS; forced expiratory volume in 1 second, $\mathbf{F E V}_{\mathbf{1}}$; forced vital capacity, FVC; odds ratio, OR; analysis of variance, ANOVA; Preserved Ratio Impaired Spirometry, PRISm; body mass index, BMI; European Respiratory Society, ERS

Funding Support: This work was supported by NHLBI R01 HL089897 and R01 HL089856. The COPDGene ${ }^{\circledR}$ study (NCT00608764) is also supported by the COPD Foundation through contributions made to an Industry Advisory Board comprised of AstraZeneca, BoehringerIngelheim, Novartis, Pfizer, GlaxoSmithKline, Siemens and Sunovion.

Date of Acceptance: February 21, 2018

Citation: Mamary AJ, Stewart JI, Kinney GL, et al for the COPDGene investigators. Race and gender disparities are evident in COPD underdiagnoses across all severities of measured airflow obstruction. Chronic Obstr Pulm Dis. 2018;5(3):177-184.

doi: https://doi.org/10.15326/jcopdf.5.3.2017.0145

1 Department of Thoracic Medicine and Surgery, Lewis Katz School of Medicine, Temple University Health System, Philadelphia, Pennsylvania

2 Department of Epidemiology, Colorado School of Public Health, Anschutz Medical Campus, University of Colorado, Aurora
3 Division of Pulmonary, Allergy and Critical Care Medicine, University of Alabama, Birmingham

4 Division of Pulmonary and Critical Care Medicine, Morehouse School of Medicine, Atlanta, Georgia 


\section{Address correspondence to:}

A. James Mamary, MD

Temple Lung Center

745 Parkinson Pavilion

3401 North Broad Street

Philadelphia $\mathrm{Pa}, 19140$

Phone: 215-707-3332

Email: albert.mamary@tuhs.temple.edu

\section{Keywords:}

diagnosis; spirometry; epidemiology; asthma; tobacco; smokers

\section{Introduction}

Chronic obstructive pulmonary disease (COPD) is a common chronic illness predominately caused by the inhalation of tobacco smoke. It is the third leading cause of death in the United States. ${ }^{1}$ COPD is a treatable and preventable disease characterized by chronic, progressive dyspnea, cough, significant comorbidity and early death. However, COPD remains vastly underdiagnosed. In the United States, the population characteristics of the approximately 13 million with undiagnosed COPD is not well understood. COPD, once considered a disease of white men, is recognized as increasingly prevalent among women and African American men. ${ }^{1-4}$ Health disparities (defined as a significant difference in health between populations) persist in most common diseases including COPD in the United States as well as the world. ${ }^{5,6}$ Reducing disparities requires definition of the vulnerable groups. 7

Approximately 13 million U.S. residents have received a diagnosis of COPD and an estimated 13 million more are unaware of their COPD diagnosis. ${ }^{8,9}$ Population estimates of COPD prevalence from the National Health And Nutrition Examination Survey and National Counsel for International Health data collected from 1970-2000 are derived mostly from individual self-reporting in phone and written population surveys and from administrative database reviews. ${ }^{8}$ The true prevalence of undiagnosed COPD among at risk individuals in the United States is unknown. The validity of self-reported COPD data to produce generalizable population prevalence estimates relies upon the assumptions that all nonCOPD reporters are truly COPD negative. Our aim is to define a large, cross-sectional, at-risk U.S. population of previously undiagnosed COPD identified in the COPDGene $^{\otimes}$ study. We hypothesize that health disparities in the COPD diagnosis are significantly influenced by race and gender.

\section{Materials and Methods Population Characteristics}

The study population was derived from participants in the COPDGene ${ }^{\circledR}$ Study Phase 1, a National Heart Lung and Blood Institute-funded multicenter observational study to analyze genetic susceptibility for the development of COPD. ${ }^{10}$ (Clinical trial registration no. NCT00608764). Participants were recruited at 21 centers in the United States from January 2008 to June 2011. COPDGene ${ }^{\circledR}$ Study Phase 1 met all criteria for institutional review board approval (Temple University institutional review board \#11369). Inclusion and exclusion criteria and study protocol are previously described. Briefly, participants were African American (AA) or non-Hispanic White (NHW), age 45-80 years old with at least a 10 pack-years history of cigarette smoking. Exclusion criteria included pregnancy, history of other lung disease except asthma, prior lobectomy, prior lung volume reduction, active cancer undergoing treatment, and known or suspected lung cancer. $^{10}$

\section{Clinical Characterization}

Clinical characterization upon study enrollment included pre- and post-bronchodilator spirometry, demographics and medical history. Smoking history and COPD and asthma diagnosiswas collected using the self-administered modified American Thoracic Society (ATS) Respiratory Epidemiology Questionnaire. Participants were asked if they had COPD diagnosed by a doctor or other health professional. Specifically, the history of COPD diagnosis was determined by a "yes" response to any of these 3 questions "Have you ever had COPD?", "Have you ever had emphysema?" and "Have you ever had chronic bronchitis?" A current asthma diagnosis was determined by a "yes" response to the question "Have you ever had asthma?" and "yes" to the question "do you still have it?" Each "yes" response was followed with, "Was it diagnosed by a doctor or other health professional?" Each participant underwent pre-bronchodilator and post-bronchodilator spirometry using an EasyOne spirometer (WelchAllyn Switzerland GmbH; Vaud, Switzerland). Predicted values were obtained using National Health and Nutritional Examination Survey III data. COPD severity (Global initiative for chronic Obstructive Lung Disease [GOLD] stage $^{1}$ ) was assigned to post- 
bronchodilator spirometry using published criteria in which airflow obstruction is defined as forced expiratory volume in 1 second $\left(F^{2} V_{1}\right)$ to forced vital capacity (FVC) ratio less than 0.7 with GOLD Stages: (1)FEV $1 \geq 80 \%$ predicted, (2)FEV $1 \geq 50 \%$ and $<80 \%$, (3) $\mathrm{FEV}_{1} \geq 30 \%$ and $<50 \%$ and $(4) \mathrm{FEV}_{1}<30 \%$.

\section{Undiagnosed COPD}

Participants in COPDGene ${ }^{\circledR}$ were recruited based on a reported $\geq 10$ pack-years smoking history, but no history of spirometry was required leaving the results of that smoking exposure open. GOLD stage was used to characterize their pulmonary health and this assessment identified undiagnosed obstructive disease in many cases. Participants were therefore classified as "known COPD" if they reported a past physician's diagnosis or "undiagnosed COPD" if they met the GOLD criteria for obstructive disease but did not report a prior physician's diagnosis of COPD.

\section{Statistical Analysis}

The effects of race and measured GOLD stage of airflow obstruction on the likelihood of undiagnosed COPD were analyzed. We also evaluated the 3-way interaction of race, gender and GOLD stage on the likelihood of undiagnosed COPD. These data were analyzed using logistic regression and trend analysis was applied to odds ratios (ORs) across GOLD stages. Descriptive data were compared by race and gender using T-test, Chi square, analysis of variance (ANOVA) and multivariate ANOVA using SAS 9.3 statistical software.

\section{Results}

Of the total 10,192 participants enrolled in the COPDGene ${ }^{\circledR}$ Study Phase 1 with a $\geq 10$ pack-years cigarette smoking burden, 8872 had normal or reduced $F E V_{1} / F V C$ ratio on enrollment spirometry. Because of the methodological limitations of using spirometry alone to diagnose COPD, it was necessary to exclude 1248 with Preserved Ratio Impaired Spirometry (PRISm) defined as FEV $1 / F V C \geq 70 \%$, $\mathrm{FEV}_{1}<80 \%$ indicating restrictive physiology and 72 with incomplete data. Fixed airflow obstruction was identified in 4484 of the 8872 on post- bronchodilator spirometry. A novel COPD diagnosis was made in 1450 individuals representing $14.3 \%$ of the entire COPDGene ${ }^{\circledast}$ Study Phase 1 cohort. (Figure 1)

Baseline demographic characteristics on this study's
8872 participants and characteristics according to race are presented in Table 1 . Participants were $45 \%$ female and $32 \%$ AA with mean age $59.9 \pm 9.1$ years, pack-year smoking burden $44.5 \pm 25.1$, and body mass index(BMI) 28.4 \pm 6.0 . In addition, 51\% were current smokers. AA participants were younger, more likely to be male and more likely to be current smokers.

\section{Figure 1. Flow Diagram of the Selected Study Population}

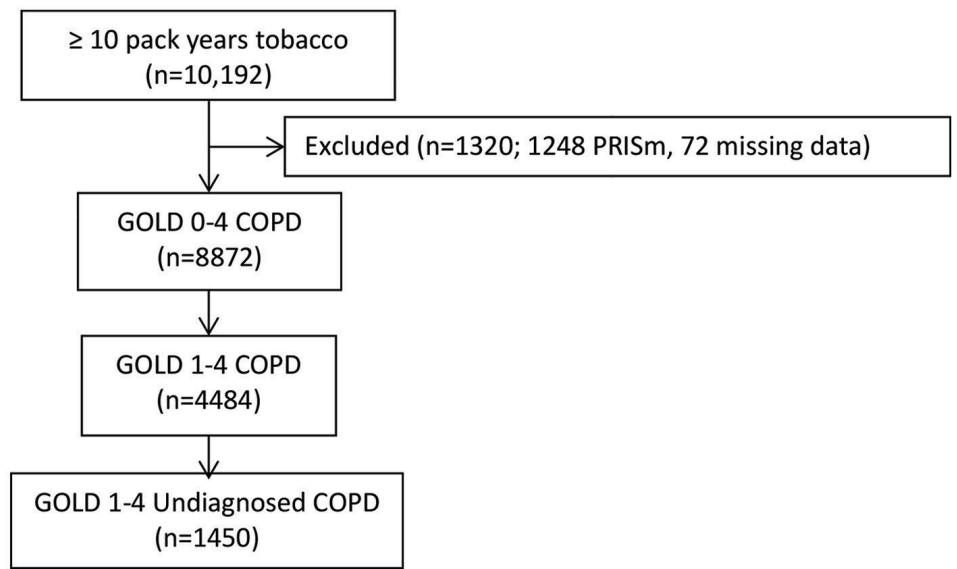

PRISm=Preserved Ratio Impaired Spirometry; GOLD=Global initiative for chronic Obstructive Lung Disease

\section{Table 1. Demographics and Clinical Characteristics on Study Admission}

\begin{tabular}{|c|c|c|c|c|}
\hline Variables & $\begin{array}{c}\text { Study } \\
\text { Participants } \\
(\mathrm{n}=8872)\end{array}$ & $\underset{(n=2823)}{\text { AA }}$ & $\begin{array}{c}\text { NHW } \\
(n=6059)\end{array}$ & $\boldsymbol{p}$ \\
\hline Female, sex, n (\%) & $4043(45)$ & $1231(14)$ & $2812(46.5)$ & 0.01 \\
\hline Age, $y r$, mean \pm SD & $59.9 \pm 9.1$ & $54.8 \pm 7.34$ & $62.2 \pm 8.9$ & $<0.0001$ \\
\hline Body Mass Index & $28.4 \pm 6.0$ & $28.6 \pm 6.3$ & $28.3 \pm 2.8$ & 0.05 \\
\hline Pack Years & $44.5 \pm 25.1$ & $38.4 \pm 21.6$ & $47.3 \pm 26.1$ & $<0.0001$ \\
\hline Current Smoker, $\mathrm{n}(\%)$ & $4563(51)$ & 2242 (79.4) & $2321(38.3)$ & $<0.0001$ \\
\hline GOLD Stages 1-4 & $4484(51)$ & $1016(36.0)$ & $3468(57.2)$ & \\
\hline FEV 1 , post-BD, & $57.4 \pm 22.8$ & & & \\
\hline
\end{tabular}

$\%$ of predicted,

GOLD 1-4

No COPD, $\mathrm{n}(\%)$

\begin{tabular}{|c|c|c|c|}
\hline \multicolumn{4}{|c|}{ Severity Previously Undiagnosed COPD } \\
\hline & $n=1450$ & $\begin{array}{c}A A \\
(n=442)\end{array}$ & $\begin{array}{c}\text { NHW } \\
(n=1008)\end{array}$ \\
\hline GOLD Grade $\mathbf{1}, \mathrm{n}$ & 548 & 138 & 410 \\
\hline GOLD Grade 2, n & 750 & 241 & 509 \\
\hline GOLD Grade $3, \mathrm{n}$ & 139 & 57 & 82 \\
\hline GOLD Grade 4, n & 13 & 6 & 7 \\
\hline
\end{tabular}

$\mathrm{AA}=$ African-American; NHW=non-Hispanic-white; GOLD=Global initiative for chronic Obstructive Lung Disease; $\mathrm{FEV}_{1}=$ forced expiratory volume in 1 second; post-BD=post-bronchodilator 
Additionally, AA participants had less total smoking burden (pack years) and a slightly higher average BMI.

A total of 1450 (32\%) of 4484 participants with objective airflow obstruction had undiagnosed COPD. This undiagnosed group represents $14.3 \%$ of the total $(10,120)$ COPDGene ${ }^{\circledR}$ Phase 1 cohort. Previously undiagnosed COPD by actual severity of airflow obstruction is displayed in Table 1. Most undiagnosed participants had mild to moderate COPD. However, 152 of the undiagnosed participants had severe GOLD stage 3 or stage 4 COPD. The prevalence of undiagnosed COPD by actual GOLD stage and race is shown in Table 1. A total of $44 \%$ of AA participants with COPD were previously undiagnosed versus $29 \%$ of NHWs. AA participants were significantly more likely to be undiagnosed versus NHWs at every measured COPD severity. (Figure 2) Trend analysis shows increasing odds of AAs having undiagnosed COPD at greater degrees of actual COPD severity (linear trend statistic $p<0.001$ ). Regardless of race, men were more likely than women to be undiagnosed with COPD at all stages of actual COPD severity. Additionally, AAs were less likely to be previously diagnosed with COPD in the cohort with GOLD stage 0 COPD (normal spirometry) (OR 1.51[1.3-1.8]) and in the 1248 PRISm participants (OR 1.8 [1.4-2.31]) who were not otherwise included. Three-way interaction of race, gender and GOLD stage was not significant indicating that both race and gender are important indicators of undiagnosed COPD but that these effects are additive. (Figure 3 )

To investigate a potential cause of undiagnosed COPD we considered self-reported current diagnosis of asthma without a COPD diagnosis. A current asthma diagnosis made by a physician or other medical professional was reported by $20 \%$ (290) of the 1450 undiagnosed COPD participants. A current asthma diagnosis without a COPD diagnosis occurred in $32 \%$ of AAs versus $16 \%$ of NHWs with undiagnosed COPD. The characteristics of these participants reporting a current asthma diagnosis without a prior COPD diagnosis are displayed by race and measured fixed airflow obstruction severity (GOLD stage COPD) in Table 2. Current asthma diagnosis in lieu of a COPD diagnosis was reported more often by AA participants, who were younger, similarly obstructed and less likely to be currently smoking than NHWs. Cumulative pack-year smoking burden was substantial and equivalent among AAs and NHWs (36.2 \pm 22.3 versus $34.4 \pm 21.1 ; p=0.5)$.

\section{Figure 2. Association of Race and Undiagnosed COPD by GOLD Grade}

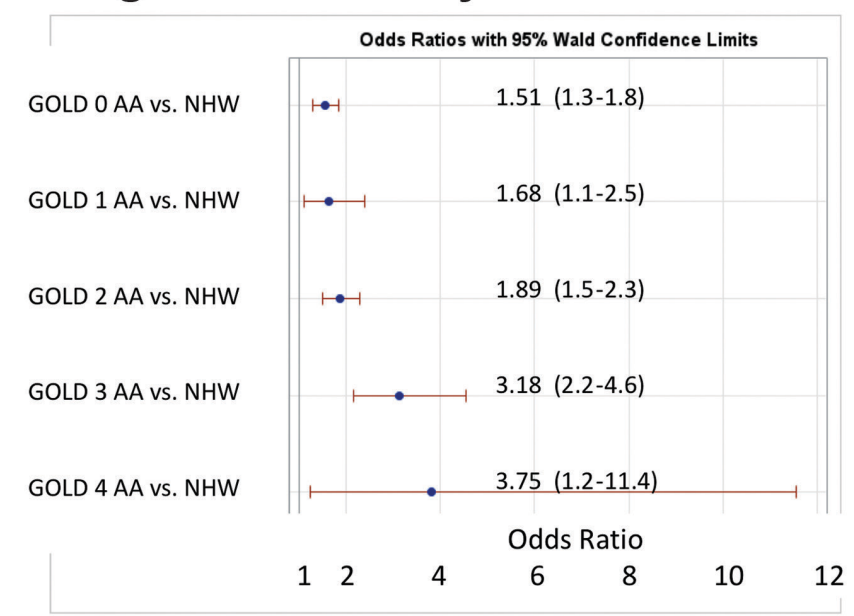

This figure provides a 2-way analysis of race and GOLD grade. AAs are at higher risk for not knowing they have COPD at every GOLD grade of airflow obstruction.

$\mathrm{AA}=\mathrm{African}$-American; NHW=non-Hispanic-white; $\mathrm{GOLD}=\mathrm{Global}$ initiative for chronic Obstructive Lung Disease

\section{Figure 3. Association of Gender and Undiagnosed COPD by Race and GOLD Grade: A Three-way Analysis}

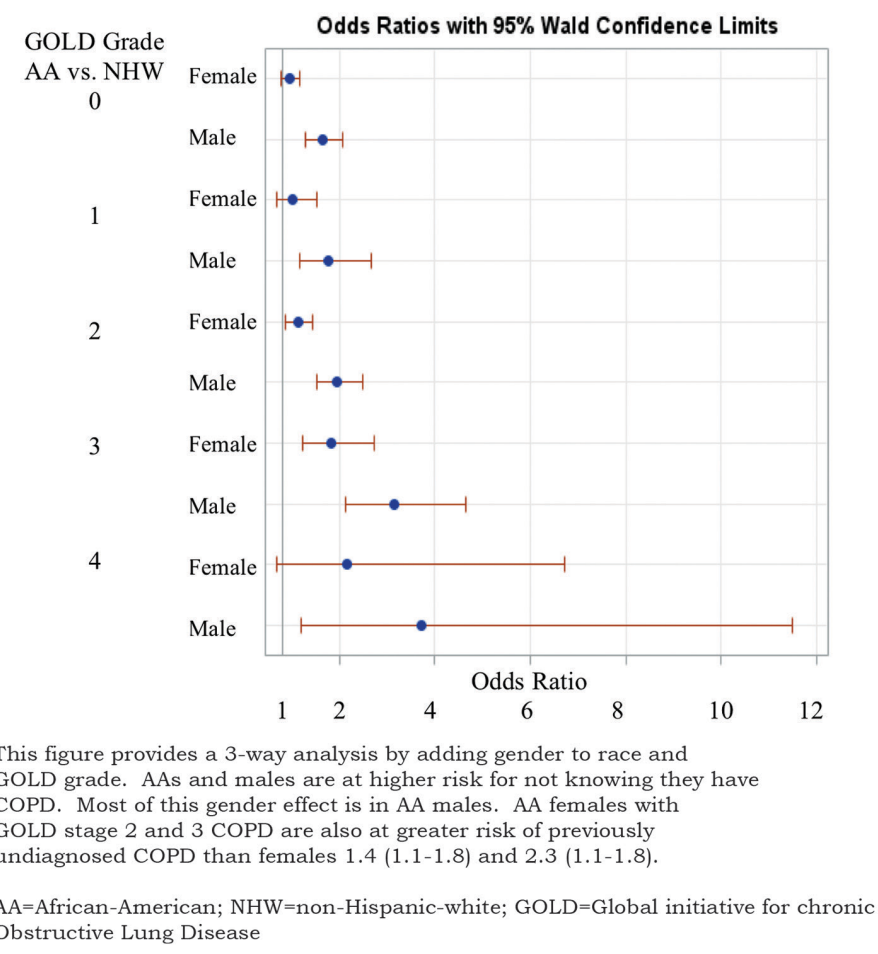
Obstructive Lung Disease

\section{Discussion}

In this study's population, the majority of undiagnosed COPD was mild or moderate in severity. Early identification may become increasingly important 


\section{Table 2. Characteristics of Study Participants with Pre-enrollment Asthma and No COPD Diagnosis}

\begin{tabular}{|c|c|c|c|}
\hline Variables & $\begin{array}{c}\mathbf{A} \mathbf{A} \\
(n=158)\end{array}$ & $\begin{array}{c}\text { NHW } \\
(n=132)\end{array}$ & $p$ \\
\hline Female, sex, n (\%) & $104(65.8)$ & $91(68.9)$ & 0.6 \\
\hline Age, yr, mean \pm SD & $53.9 \pm 1.5$ & $60.9 \pm 9.0$ & $<0.0001$ \\
\hline Body Mass Index & $30.5 \pm 7.0$ & $30.0 \pm 6.6$ & 0.5 \\
\hline Pack Years & $36.2 \pm 22.3$ & $34.4 \pm 21.1$ & 0.5 \\
\hline Current Smoker, n (\%) & $36(22.8)$ & $93(70.5)$ & $<0.0001$ \\
\hline FEV 1, post-BD, & $2.1 \pm 0.7$ & $2.3 \pm 0.7$ & 0.001 \\
\hline
\end{tabular}

Liters \pm SD, GOLD 1-4

\begin{tabular}{|c|c|c|c|}
\hline \multicolumn{4}{|l|}{ COPD Severity } \\
\hline & $\begin{array}{c}\text { AA } \\
(n=158)\end{array}$ & $\begin{array}{c}\text { NHW } \\
(n=132)\end{array}$ & $\begin{array}{c}\text { Total } \\
(n=290)\end{array}$ \\
\hline GOLD $0, \mathrm{n}(\%)$ & 81 (51.3) & $66(50.0)$ & 147 (50.7) \\
\hline GOLD $1, \mathrm{n}(\%)$ & $15(9.5)$ & $23(17.4)$ & 38 (13.1) \\
\hline GOLD 2, n (\%) & $47(29.7)$ & $33(25)$ & 80 (27.6) \\
\hline GOLD 3, n (\%) & $14(8.9)$ & $9(6.8)$ & $23(7.9)$ \\
\hline GOLD 4, n (\%) & $1(0.6)$ & $1(0.76)$ & $2(0.69)$ \\
\hline
\end{tabular}

GOLD Grade COPD by post-BD spirometry at study enrollment.

$\mathrm{AA}=$ African-American; NHW=non-Hispanic-white; GOLD=Global initiative for chronic Obstructive Lung Disease; $\mathrm{FEV}_{1}=$ forced expiratory volume in 1 second; post- $\mathrm{BD}=$ post-bronchodilator

for patients to benefit maximally from emerging COPD therapies. Identifying population disparities in undiagnosed COPD is an opportunity to understand and change unevenly applied medical care. The U.S. Department of Health and Human Service's "Healthy People 2020" initiative ${ }^{11}$ and the ATS/ European Respiratory Society(ERS) official policy statement: "Disparities in Respiratory Health,"7 serve as a commitment and framework to define and reduce respiratory health disparities: "The vision of the ATS and ERS is that all persons attain better and sustained respiratory health."7

To our knowledge the present study is the first prospective characterization of a geographically and demographically diverse group of AAs, NHWs, and male and female current and former smokers. We found substantial, undiagnosed COPD in the entire cohort. However, the risk for undiagnosed COPD was not uniform within the study population with significant disparity by race and gender.

Within our study's cohort of individuals identified with fixed airflow obstruction, males were less likely to have been previously diagnosed with COPD. This effect is primarily determined by the underdiagnosis of AA males. The underdiagnosis of COPD in males is an unanticipated finding considering historical attitudes and published data. Historically, COPD was thought to be more prevalent among men than women. These antiquated attitudes may have contributed to historical physician bias toward higher recognition and diagnosis of COPD in men versus women with similar symptoms and smoking histories. In a 2001 survey of a random sample of 192 U.S. primary care physicians using a hypothetical case presentation, male patients were more likely to be given a COPD diagnosis. This recognition bias was mitigated by the addition of spirometry data. ${ }^{12}$ Two large foreign studies of spirometry testing in randomly selected individuals $\geq 40$ years of age, have shown high prevalence of undiagnosed COPD representing all levels of measured airflow obstruction. ${ }^{13,14}$ In the Polish study there was no gender disparity in undiagnosed COPD in current or former smokers. ${ }^{13}$ The Spanish study suggested that women were at greater risk of undiagnosed COPD with a subgroup of older men ( $>60$ years) who are urban dwellers and more educated with a greater than 15 pack-years smoking burden having the highest probability of having a prior diagnosis of COPD. ${ }^{14}$ However, no historical data exists for undiagnosed COPD in at risk populations in the United States. Our findings suggest that men, rather than women, are at higher risk of COPD underdiagnosis. These data demonstrate gender disparity in COPD diagnosis with higher rates of undiagnosed COPD in men, regardless of race. Gender differences in symptom perception and attitudes toward medical care may contribute to these observations. It has also been previously shown that women generally have more dyspnea at a lesser airflow obstruction and this is hypothesized to be due to smaller inspiratory capacity related to lower thoracic volumes. ${ }^{15}$ Women report symptoms earlier and more frequently and generally utilize more health care resources than men. ${ }^{16}$ Enhanced symptom reporting may contribute to earlier diagnosis and may explain the lower observed rates of undiagnosed COPD in women in COPDGene ${ }^{\circledR}$.

Within our study's cohort, looking at measured airflow obstruction at study entry, AAs were less likely than NHWs to be previously diagnosed with COPD. This racial disparity persisted with increasing odds of underdiagnosis at successive severities of measured airflow obstruction. The undiagnosed AA cohort was 
younger, with more current smoking and fewer total pack years of smoking burden.

Important social and economic factors drive human health disparities. ${ }^{17,18}$ The 2010 U.S. census survey ${ }^{19}$ lists the total U.S. population as 308.7 million, with $80.7 \%$ of the population classified as urban, 38.9 million (14\%) as AA or Black alone and 196.8 million (63.7\%) as NHW. AAs are twice as likely as NHWs to be classified as living in poverty--25.8\% versus $11.6 \%$ respectively--compared to $14 \%$ of the entire U.S. population. This study's participants are from the 21 COPDGene ${ }^{\circledast}$ enrollment sites located in U.S. population centers along the seaboards, great lakes and rivers. COPDGene ${ }^{\circledR}$ enrolled patients from existing pulmonary practices at enrollment centers and through advertisement-based recruiting.

Our findings of racial disparity in COPD diagnosis are consistent with some larger themes of respiratory health in the United States. Several U.S. studies document disparity in the application of standard of care interventions in COPD known to decrease mortality and morbidity including smoking cessation programs, influenza vaccination and supplemental oxygen. ${ }^{6}$ Tobacco smoke, air pollution, occupational and environmental inhalational exposures occur disproportionally in ethnic minorities and individuals with lower socioeconomic status. Differential access to quality health care is common with important known determinants such as cost, employment-based health insurance, physician availability, transportation, education and social attitudes and behavior. ${ }^{17,18}$ The monetary cost of undiagnosed COPD is high and is primarily driven by increased hospitalization costs, not the cost of diagnosis and chronic treatment. ${ }^{20}$ Historically, AAs are more likely than white individuals to receive emergent COPD treatment versus officebased care. $^{8}$

Regardless, diagnostic spirometry is an effective, easy to perform, low cost, office-based test that is underutilized in patients at risk for COPD. ${ }^{21}$ The ATS, ERS, Centers for Disease Control and Prevention and GOLD all recommend spirometry to diagnose COPD in at risk patients, those with respiratory symptoms and smoking history. 1,7,17 Anthonisen et al documented wide-spread physician underuse of diagnostic spirometry in patients coded with a clinically-derived diagnosis of COPD. ${ }^{22,23}$ History and physical exam are not reliably sensitive to exclude fixed airflow obstruction. Spirometry is not uniformly available as an office-based tool in primary care settings. The additional step of off-site testing and specialist referral add additional barriers to the diagnosis of COPD.

It is known that AAs with severe COPD have more severe airflow obstruction at younger ages with less pack-year smoking burdens than whites. ${ }^{24}$ However, our data do not support earlier and more frequent COPD diagnosis among AAs.

In the present study, an asthma diagnosis exclusive of a COPD diagnosis was observed in participants with fixed airflow obstruction and likely contributed to the disparity we observed in COPD diagnosis. A significant subgroup of those with undiagnosed COPD carried a diagnosis of asthma at study enrollment. In this subgroup with fixed airflow obstruction, AAs were more likely diagnosed with asthma and not COPD than NHWs (RR: 2.66). This pattern was seen increasingly in participants with moderate or severe fixed airflow obstruction (RR: 3.3). The differentiation of true asthma with fixed airflow obstruction versus COPD is not possible in our participants, all of whom had a significant burden of smoking. The incorrect or exclusive diagnosis of asthma may contribute to the underdiagnosis of COPD especially in AAs.

\section{Limitations}

Ascertainment bias may limit all cross-sectional population studies. The present study mitigates the potential bias through a large number of participants and enrolling centers from across the country. Enrollment pathway data was not collected. Additionally, participants may under-report previously diagnosed COPD for various reasons including denial, poor communication by prior physicians and real or perceived effects on employment and insurance premiums. We regard the study enrollment pathway and self-reporting of COPD diagnosis as a product of real patient behavior and experience and the disparities that exist in access to and delivery of health care in the United States. The paradigm of an $\mathrm{FEV}_{1} / \mathrm{FVC}$ ratio less than 0.7 as the sine qua non of COPD excludes participants who may have radiographic evidence of COPD and normal spirometry or PRISm.

\section{Conclusion}

COPDGene ${ }^{\circledR}$ provides a unique opportunity to examine the prevalence of the diagnosis of COPD in a large, cross-sectional U.S. cohort. Undiagnosed COPD 
is common among individuals at least 45 years of age with a $\geq 10$ pack-years cigarette smoking burden and is found at all severities of airflow obstruction. AAs are at greater risk for undiagnosed COPD than NHWs and men are at greater risk than women. Population disparities in the diagnosis of COPD should be considered when defining COPD prevalence and addressed to improve the health of the U.S. population.

\section{Declaration of Interest}

The authors do not have financial, consulting or personal relationships with other people or organizations that could influence this work. The authors received no assistance in writing this manuscript. The authors have no personal grant funding related to this work. 


\section{References}

1. Global initiative for chronic obstructive pulmonary disease (GOLD). Global strategy for the diagnosis, management and prevention of chronic obstructive pulmonary disease. GOLD website. http://goldcopd.org/global-strategy-diagnosis-manage ment-prevention-copd-2016.

Published 2016. Accessed December 2016.

2. Mannino DM, Buist AS. Global burden of COPD: risk factors, prevalence, and future trends. Lancet. 2007;370(9589):765-773. doi: https://doi.org/10.1016/S0140-6736(07)61380-4

3. Petty TL. Scope of the COPD problem in North America. Early studies of prevalence and NHANES III Data: basis for early identification and intervention. Chest. 2000;117(5):326S-31S. doi: https://doi.org/10.1378/chest.117.5_suppl_2.326S

4. Shaya FT, Maneval MS, Confidence M, et al. Burden of COPD, asthma, and concomitant COPD and asthma among adults: racial disparities in a Medicaid population. Chest. 2009;136(2):405-11. doi: https://doi.org/10.1378/chest.08-2304

5. Camp PG, Goring SM. Gender and the diagnosis, management, and surveillance of chronic obstructive pulmonary disease. Proc Am Thorac Soc. 2007;4(8):686-691.

doi: https://doi.org/10.1513/pats.200706-081SD

6. Dransfield MT, Bailey WC. COPD: racial disparities in susceptibility, treatment, and outcomes. Clin Chest Med. 2006;27(3):463-471.

doi: https://doi.org/10.1016/j.ccm.2006.04.005

7. Schraufnagel DE, Blasi F, Kraft M, Gaga M, Finn PW, Rabe KF for the ATS/ERS Committee on Disparities in Respiratory Health. An official American Thoracic Society/European Respiratory Society policy statement: disparities in respiratory health. Am J Respir Crit Care Med. 2013;188(7):865-871.

doi: https://doi.org/10.1164/rccm.201308-1509ST

8. Mannino DM, Homa DM, Akinbami LJ, et al. Chronic obstructive pulmonary disease surveillance--United States, 1971-2000. MMWR Surveill Summ. 2002;51:1-16.

9. Ford ES, Croft JB, Mannino DM, Wheaton AG, Zhang X, Giles WH. COPD surveillance--United States, 1999-2011. Chest. 2013;144(1):284-305. doi: https://doi.org/10.1378/chest.13-0809

10. Regan EA, Hokanson JE, Murphy JR, et al for the COPDGene investigators. Genetic epidemiology of COPD (COPDGene) study design. COPD. 2010;7 (1):32-43.

doi: https://doi.org/10.3109/15412550903499522

11. U.S. Department of Health and Human Services, Office of Disease Prevention and Health Promotion. Healthy people 2020. Healthy people website. https:/www.healthypeople.gov/ Published 2014. Updated 2018. Accessed December 2016.

12. Chapman KR, Tashkin DP, Pye DJ. Gender bias in the diagnosis of COPD. Chest. 2001;119(6):1691-1695.

doi: https://doi.org/10.1378/chest.119.6.1691
13. Bednarek M, Maciejewski J, Wozniak M, Kuca P, Zielinski J. Prevalence, severity and underdiagnosis of COPD in the primary care setting. Thorax. 2008;63(5):402-407.

doi: https://doi.org/10.1136/thx.2007.085456

14. Pena VS, Miravitlles M, Gabriel R, et al. Geographic variations in prevalence and underdiagnosis of COPD: results of the IBERPOC multicentre epidemiological study. Chest. 2000;118(4):981-989. doi: https://doi.org/10.1378/chest.118.4.981

15. de Torres JP, Casanova C, Hernandez C, Abreu J, AquirreJaime A, Celli BR. Gender and COPD in patients attending a pulmonary clinic. Chest. 2005;128(4):2012-2016. doi: https://doi.org/10.1378/chest.128.4.2012

16. Ladwig KH, Marten-Mittag B, Formanek B, Dammann G. Gender differences of symptom reporting and medical health care utilization in the German population. Eur $J$ Epidemiol. 2000; 16(6):511-518. doi: https://doi.org/10.1023/A:1007629920752

17. Centers for Disease Control and Prevention (CDC). NCHHSTP Social determinants of health. CDC website. http://www.cdc.gov/nchhstp/socialdeterminants/index.html Published 2013. Updated 2017. Accessed December 2016.

18. World Health Organization (WHO). Closing the gap in a generation: health equity through action on the social determinants of health. Final eport of the Commission on Social Determinants of Health. WHO website. http://www. who.int/social_determinants/thecommission/finalreport/en/ Published 2008. Accessed December 2015.

19. United States Census Bureau. Decennial census of population, by decade: 2010 Census. U.S. Census Bureau website. https://www. census.gov/programs-surveys/decennial-census/decade.2010. html Published 2010. Accessed December 2016.

20. Mapel DW, Robinson SB, Dastani HB, Shah H, Phillips AL, Lydick E. The direct medical costs of undiagnosed chronic obstructive pulmonary disease. Value Health. 2008;11(4):628636. doi: https://doi.org/10.1111/j.1524-4733.2007.00305.x

21. Buffels J, Degryse J, Heyrman J, Decrammer M. Office spirometry significantly improves early detection of COPD in general practice: the DIDASCO study. Chest. 2004;125(4):13941399. doi: https://doi.org/10.1378/chest.125.4.1394

22. Anthonisen NR, Dik N, Manfreda J, Roos LL. Spirometry and obstructive lung disease in Manitoba. Can Respir J. 2001;8(6):421-426. doi: https://doi.org/10.1155/2001/572825

23. Anthonisen NR, Connett JE, Enright PL, Manfreda J and the Lung Health Study Research Group. Hospitalizations and mortality in the Lung Health Study. Am J Respir Crit Care Med. 2002;166(3):333-339. doi: https://doi.org/10.1164/rccm.2110093

24. Chatila WM, Wynkoop WA, Vance G, Criner GJ. Smoking patterns in African Americans and whites with advanced COPD. Chest. 2001;125(1):15-21.

doi: https://doi.org/10.1378/chest.125.1.15 